\title{
Optoelectronic and elastic response of fluorinated hexagonal boron nitride monolayer
}

\author{
Shambhu Bhandari Sharma ${ }^{a}$ \\ ${ }^{a}$ Goldengate International College, Tribhuvan University, Kathmandu, Nepal
}

\section{ARTICLE INFO}

Keywords:

SIESTA

Fluorination

Structural and electronic properties

Mechanical properties

Optical properties

\begin{abstract}
A B S TRACT
The inherited insulating behavior of hexagonal boron nitride (h-BN) monolayer restricts its application in several optoelectronic devices, so finding a technique to reduce the bandgap allows it to possess the semiconducting functionality. Here, an experimentally feasible fluorinated hexagonal boron nitride (FBNF), a structurally, dynamically, and mechanically stable monolayer is reported by using density functional theory calculations. The significant geometrical transformation from planer h-BN to buckled FBNF softens the structure by retaining the mechanical isotropy and structural symmetry. Remarkably, the induced direct bandgap semiconducting behavior after fluorination enhances the optical absorbance and reflectivity, reduces energy loss, creates strong optical anisotropy, and makes FBNF monolayer a proper material in the optoelectronic and nanomechanical applications.
\end{abstract}

\section{Introduction}

The finding of reducing the dimension from threedimensional (3D) graphite to two-dimensional (2D)[1] grap- hene suggests the further possibilities of low-dimensional materials. Since then, several 2D materials like h-BN[2, 3, 4], $\mathrm{SiC}[5]$, phosphorene[6], transitional metal dichalcogenides[7 8], MXenes[9] have been studied theoretically and experimentally. The 2D ultra-thin hexagonal boron nitride (h-BN) monolayer is getting great attention due to it's intriguing physical and chemical properties [10, 2, 3]. It has been successfully synthesized by several experimental techniques such as the ball milling method[11], highenergy electron radiation[12], chemical-solution-derived method[13], sonication centrifugation method[14], molecular beam epitaxy method[15]. It is a low-cost, highly active, and stable catalyst[16]. It shows excellent bio-mechanism, useful in scanning probe microscopes in nano-medical field[17]. It possesses excellent mechanical rigidity [18], and the second-highest thermal conductivity among all 2D semiconductors and insulators [19].

Unfortunately, the electronic bandgap of the h-BN is too large (natural insulator) that lacks the semiconducting functionality. Therefore, most of the h-BN materials are used as insulating materials[20][21] and restricts its application in several optoelectronic devices. Various approaches such as doping, vacancy defects, absorption, strain, external electric field, and chemical passivation in the pristine 2D structure [22][23][23][24] have been implemented to settle this problem. However, applying an external electric field or strain would considerably make the device configuration complicated[25]. Meanwhile, several experimental and theoretical works show that chemical passivation is one of the best approaches to tune the

\footnotetext{
*Corresponding author

@ shambhubhandari789@gmail.com (S.B. Sharma) ORCID(s):
}

structural, electronic, and optical properties of the compound. For instance, the first-principles calculations demonstrate that fluorination is a practical route to induce halfmetallicity in zigzag boron nitride nanoribbon[26]. Similarly, the passivation of hydrogen and fluorine on the h-BN monolayer changes the electronic and magnetic properties 'drastically[27]. The structural and electronic properties of graphene supported on FBNF monolayer substrate modifies the electronic properties of graphene supporting the fabrication of high-performance graphene-based nanodevices[28]. Additionally, the controlled adsorption of fluorine atoms on both sides of a pristine h-BN sheet induces flatness distortion and an out-of-plane dipole moment in a chair conformer[29]. The fluorination of electrically insulating the h-BN subsequently modifies the electronic band structure to a wide bandgap magnetic semiconductor via the introduction of defect levels. The electrophilic nature of fluorine causes changes in the charge distribution around neighboring nitrogen atoms in the h-BN, leading to room temperature weak ferromagnetism[30]. Very recently, an experimental study shows that exfoliation of the h-BN sheets from bulk material and surface treatment by fluorination makes structural improvements that lead to enhancement in electrical and optical properties[31].

Multiple theoretical and experimental investigations have been conducted to study various features of pure and passivated the h-BN monolayer, what is lacking here is a meticulous study on the structural, mechanical, and optoelectronic response of fluorinated h-BN. Intrigued by the prior findings, the first-principles calculations are performed with the density functional theory to investigate and unravel the underlined properties of fluorinated hexagonal boron nitride monolayer.

\section{Computational Details}

The density functional theory (DFT) implemented in the Spanish initiative for electronic simulations with thousands 
of atoms (SIESTA) [32, 33] with norm-conserving pseudopotentials in the semilocal form [34] is used for the calculations. The employed generalized gradient approximation (GGA) functional with Perdew-Burke-Ernzerhof (PBE) [35] treats underlying exchange and correlation within the double zeta plus polarization (DZP) basis sets. The K points $20 \times 20 \times 1$ of Monkhrost pack scheme [36] is used to perform Brillouin zone integration. The reciprocal space is expanded by using an energy cutoff of 350 Rydberg. The atomic relaxation is achieved when the force reached the value of 0.02 eV/Å using the standard conjugate-gradient (CG) technique. The convergence criteria for the energy of the self-consistent field is set to $1.0 \times 10^{-6} \mathrm{eV}$. The vacuum gap of $25 \AA$ is used along the z-direction to prevent unnecessary interactions between the adjacent unit cells. The chemical stability is achieved by calculating and analyzing the formation $\left(E_{f}\right)$ and cohesive $\left(E_{c o h}\right)[29]$ energies.

$$
\begin{aligned}
& E_{\mathrm{coh}}=\left(E-\sum_{\mathrm{i}=\mathrm{B}, \mathrm{N}, \mathrm{F}} n_{\mathrm{i}} E_{\mathrm{i}}\right) / N \\
& E_{f}=\frac{1}{N}\left(E_{t}-E_{\mathrm{BN}}-n_{F_{2}} E_{F_{2}}\right)
\end{aligned}
$$

where $E_{t}, E_{B N}$ and $E_{F_{2}}$ represents is the total energy of FBNF, h-BN and $F_{2}$ molecule respectively. The $n_{F_{2}}$ and $N$ denotes the number of fluorine molecule and total number of atoms. Similarly, in cohesive energy expression, the $E$ is total energy of the preferred system having $\mathrm{N}$ atoms. $E_{i}$ represents the total energy of an isolated $\mathrm{i}(\mathrm{B}, \mathrm{N}, \mathrm{F})$ atom and $n_{i}$ is the number of a specific atom i per unit cell. The frozen phonon method implemented in the Vibra package of SIESTA is adopted for the phonon dispersion calculation. An accurate force matrix is achieved at $5 \times 5 \times 1$ supercell of convergence. For optical properties calculations, a denser k-point mesh, i.e. $60 \times 60 \times 1$, within the Monkhorst-Pack scheme and optical broadening of $0.1 \mathrm{eV}$ is used. Optical calculations in SIESTA are based on the first order time dependent perturbation theory (TDP)[37]. The complex dielectric function $(\varepsilon(\omega))$ can be expressed as:

$$
\varepsilon(\omega)=\varepsilon_{1}(\omega)+i \varepsilon_{2}(\omega)
$$

where $\varepsilon_{2}(\omega)$ is imaginary part of $\varepsilon(\omega)$ and can be obtained with the help of TDP [38]

$$
\begin{array}{r}
\varepsilon_{2}(\omega)=\frac{e^{2}}{\omega^{2} \pi m^{2}} \sum_{v, c} \int_{B Z} d \vec{k}\left|\left\langle\psi_{c k}|\hat{e} \cdot \vec{p}| \psi_{v k}\right\rangle\right|^{2} \delta\left(E_{c}(k)-\right. \\
\left.E_{v}(k)-\hbar \omega\right)
\end{array}
$$

Here, $v$ and $\mathrm{c}$ represent the valence and the conduction band states, respectively. $E_{(c, v)}(k)$ and $\psi_{(c, v), k}$ are the corresponding energy and eigenfunction of these states. $\vec{p}$ and $\hat{e}$ are the momentum operator and polarization vector, respectively. The equation (4) displays the connection between optical and electronic properties. Further, real part of dielectric fuction $\varepsilon_{1}(\omega)$ is obtained by Kramer-Kronig transformation (KK) of $\varepsilon_{2}(\omega)$ and is expressed as:

$$
\varepsilon_{1}(\omega)=1+\frac{2}{\pi} P \int_{0}^{\infty} \frac{\varepsilon_{2}\left(\omega^{\prime}\right) \omega^{\prime}}{\omega^{\prime 2}-\omega^{2}} d \omega^{\prime}
$$

where $P$ denotes the principle part of $\varepsilon_{1}(\omega)$ [38].

Further, the complex refractive index $(N)$ is expressed as $N=\sqrt{\varepsilon(\omega)}=\eta(\omega)+i K(\omega)$, where $\eta(\omega)$ and $K(\omega)$ are the refractive index and extinction coefficient, respectively. These parameters are expressed as:

$$
\begin{aligned}
& \eta(\omega)=\left(\frac{\sqrt{\varepsilon_{1}^{2}(\omega)+\varepsilon_{2}^{2}(\omega)}+\varepsilon_{1}(\omega)}{2}\right)^{\frac{1}{2}} \\
& K(\omega)=\left(\frac{\sqrt{\varepsilon_{1}^{2}(\omega)+\varepsilon_{2}^{2}(\omega)}-\varepsilon_{1}(\omega)}{2}\right)^{\frac{1}{2}}
\end{aligned}
$$

Further, the reflectivity, $\mathrm{R}(\omega)$ and absorption coefficient, $\alpha(\omega)$ are expressed as [39]:

$$
\begin{aligned}
& R(\omega)=\frac{K(\omega)^{2}+[1-n(\omega)]^{2}}{K(\omega)^{2}+[1+n(\omega)]^{2}} \\
& \alpha(\omega)=\frac{2 \omega K(\omega)}{c}
\end{aligned}
$$

Additionally, the electron energy loss function, $\mathrm{L}(\omega)$ is given by the relation $L(\omega)=\operatorname{Im}\left(-\frac{1}{\varepsilon(\omega)}\right)$ and also in terms of $\varepsilon_{1}(\omega)$ and $\varepsilon_{2}(\omega)$,

$$
L(\omega)=\frac{\varepsilon_{2}(\omega)}{\varepsilon_{1}(\omega)^{2}+\varepsilon_{2}(\omega)^{2}}
$$

All these optical parameters are calculated in the interval between 0 to $25 \mathrm{eV}$ for in-plane $(\mathrm{E} \| \mathrm{x})$, and out-of-plane polarization $(\mathrm{E} \| \mathrm{z})$ of electric field.

\section{Results and Discussion}

\subsection{Structural properties}

The optimized unit cell of pristine h-BN has planer geometry with lattice constant $2.52 \AA$ and $\mathrm{sp}^{2}$ hybridized $\mathrm{B}-\mathrm{N}$ bond of length $1.45 \AA$. By adding $\mathrm{F}$ atoms on top of $\mathrm{B}-$ sites and bottom of $\mathrm{N}$-sites (Fig. 1) in the unit cell of h-BN, the FBNF monolayer is designed which is allowed to fully relax until it achieves the favorable stable configuration. The optimized structure is buckled $(0.50 \AA)$ with lattice constant $2.66 \AA$ and $\mathrm{sp}^{3}$ hybridized longer $\mathrm{B}-\mathrm{N}$ bond of length $1.62 \AA$. The details of bond lengths in optimized structures are presented in Table 1. The lattice constant of FBNF is $10 \%$ more than pristine structure however retains the hexagonal symmetry similar to fluorinated graphene [40] 


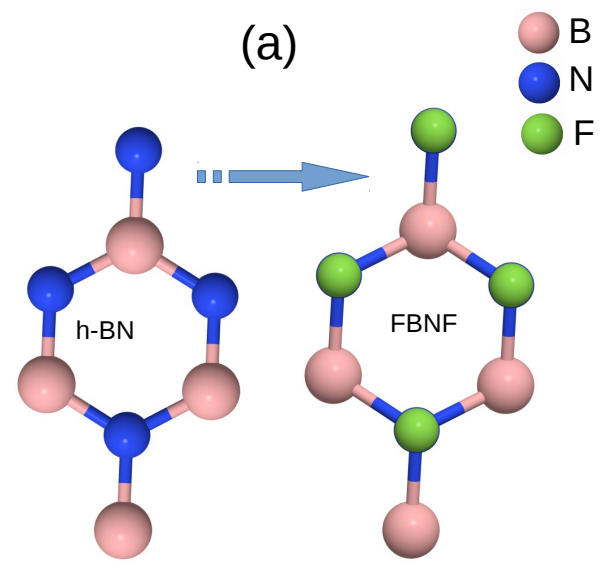

(b)

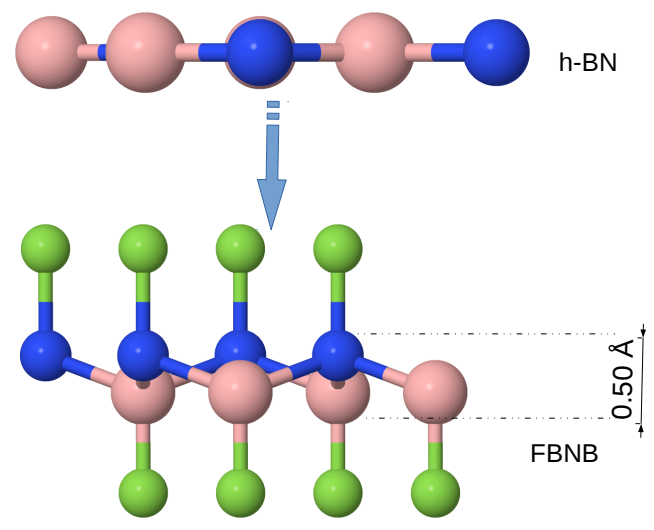

Figure 1: (Color online) Optimized strucutre $(2 \times 2 \times 1$ supercell) of $h-B N$ and FBNF monolayer (a) top view (b) side view.

and $\mathrm{SiC}[41]$. This increment is attributed to the larger radius of the $\mathrm{F}$ atom and inter-atomic repulsion. The structural stability is confirmed by calculating the cohesive energy (Equ. 1) which is $-6.91 \mathrm{eV} /$ atom and $-4.83 \mathrm{eV} /$ atom for h-BN and FBNF monolayer, respectively. The relatively smaller negative cohesive energy in FBNF is connected to the longer $\mathrm{B}-\mathrm{N}$ bonding. The formation energy (Equ. 2) of FBNF is $-0.65 \mathrm{eV} /$ atom which confirms the experimental feasibility of flourination. These findings are very consistent with the previous theoretical and experimental results[42][43][44][45][46].

The nature of chemical bonds are analyzed by Mulliken charge density calculations (Fig. 2(a)-(f)). The red to pink color codes (Fig. 2(c) indicates the lowest to highest charge density distribution. The considerable overlap of electronic wave functions (Fig. 2(d) with sharing of the electron among $\mathrm{B}$ and $\mathrm{N}$ atoms in FBNF monolayer reveals the presence of covalent $\mathrm{B}-\mathrm{N}$ bonding, which agrees with the previous report[45]. A similar configuration is found between $\mathrm{F}$ and $\mathrm{B}$ atoms (Fig. 2f) thereby confirms the covalent F-B bonding. On the other hand, there is a small distortion of the overlapped wave function at the center of $\mathrm{F}$ and $\mathrm{N}$ atoms ( Fig. 2(e)) demonstrates a partly ionic and covalent F-N
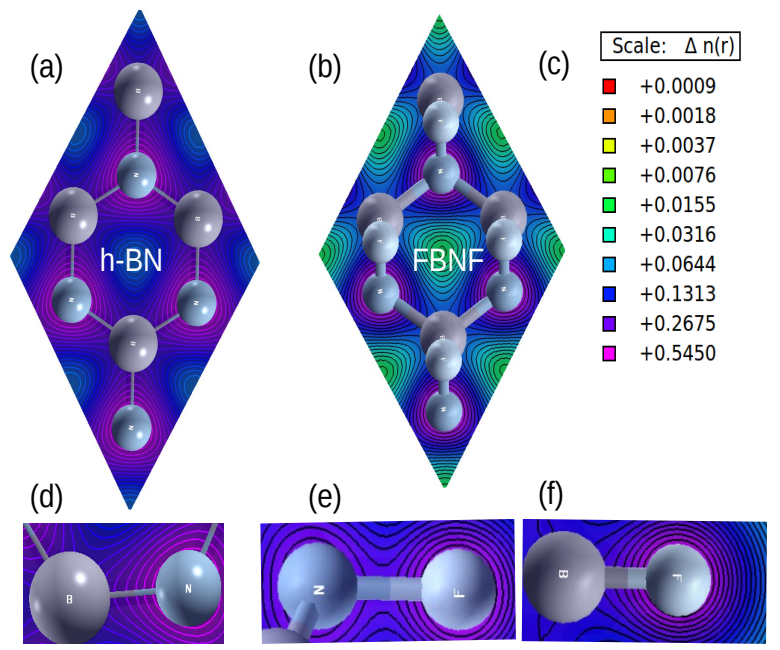

Figure 2: (Color online) Valence charge density contour plot of (a) h-BN (b) FBNF (d)-(e) Zoomed view of charge density between respective atoms (c) charge density index.

bonding. Further, to test the dynamic stability the phonon dispersion calculations are performed (Fig. 3(a) and (b)) along $\Gamma-\mathrm{K}-\mathrm{M}-\Gamma$ high-symmetry lines. The presence of real frequencies in both monolayers demonstrates good kinetic and dynamical stability.

After confirming the structural, and dynamical stability, it's crucial to test the mechanical stability and strength of the material because the strain is unavoidable in material growth and processing [47]

\subsection{Mechanical properties}

The h-BN monolayer, also known as "white graphene", has extremely comparable physical and chemical properties with graphene [18]. Particularly, the $s p^{2}$ hybridized covalent bonds between $\mathrm{C}$ atoms in graphene and $\mathrm{B}-\mathrm{N}$ bonding in h-BN makes both materials mechanically strong and stable. However, the fluorination in $\mathrm{h}-\mathrm{BN}$ makes the $\mathrm{B}-\mathrm{N}$ bonding $s p^{3}$ hybridized, which distorts the planar geometry[29], the mechanical response of fluorinated h-BN monolayer, FBNF, is interesting to study.

To calculate the mechanical parameters, the strain is applied in the $\mathrm{x}$-axis $\left(\varepsilon_{x}\right), \mathrm{y}$-axis $\left(\varepsilon_{y}\right)$, and in both directions $\left(\varepsilon_{x} \varepsilon_{y}\right)$ of the monolayer. The strain is applied from $-2 \%$ to $2 \%$ for each direction with an increment of $1 \%$. For each iteration of strain, the atomic positions of the system is fully relaxed. Further, the result is fitted to the strain energy, $E_{s}=a_{1} \varepsilon_{x}^{2}+a_{2} \varepsilon_{y}^{2}+a_{3} \varepsilon_{x} \varepsilon_{y}$ [50]. Here, $a_{1}, a_{2}$ and $a_{3}$ are three constants that are obtained during the fitting process. Due to the isotropy in the honeycomb symmetry, $a_{1}$ is equal to $a_{2}$. The 3D surface fit (Fig. 4) shows the distribution of strain energy with respect to the different possible value of applied strain. The maximum strain energy value is lower in FBNF predicting the relatively smaller mechanical strength. The preceding expression is also obtained from elasticity matrix in terms of elastic stiffness constants $C_{11}$ and $C_{12}$ 
Table 1

Summary of structural and electronic properties: lattice constant a, bond length $d_{X Y}$ where, $(\mathrm{X}, \mathrm{Y}=\mathrm{B}, \mathrm{N}, \mathrm{F})$, bandgap $\mathrm{E}_{g}$, cohesive energy $E_{c o h}$, formation energy $E_{f}$

\begin{tabular}{|c|c|c|c|c|c|c|c|}
\hline Monolayer & $\begin{array}{l}a \\
(\AA)\end{array}$ & $d_{B N}$ & $\begin{array}{r}d(\AA) \\
d_{N F}\end{array}$ & $d_{B F}$ & $\begin{array}{l}E_{g} \\
(\mathrm{eV})\end{array}$ & $\begin{array}{l}E_{c o h} \\
(\mathrm{eV})\end{array}$ & $\begin{array}{l}E_{f} \\
(\mathrm{eV})\end{array}$ \\
\hline h-BN & 2.52 & 1.45 & - & - & 4.62 & -6.91 & - \\
\hline Ref. & $2.51[48]$ & $1.45[48]$ & - & - & $4.63[48]$ & $-7.09[49]$ & - \\
\hline FBNF & 2.66 & 1.62 & 1.45 & 1.36 & 3.39 & -4.83 & -0.66 \\
\hline \multirow[t]{2}{*}{ Ref.[29] } & - & 1.62 & 1.44 & 1.35 & 3.30 & -4.96 & -0.65 \\
\hline & - & - & $1.42[28]$ & $1.34[28]$ & $2.80[28]$ & - & - \\
\hline
\end{tabular}

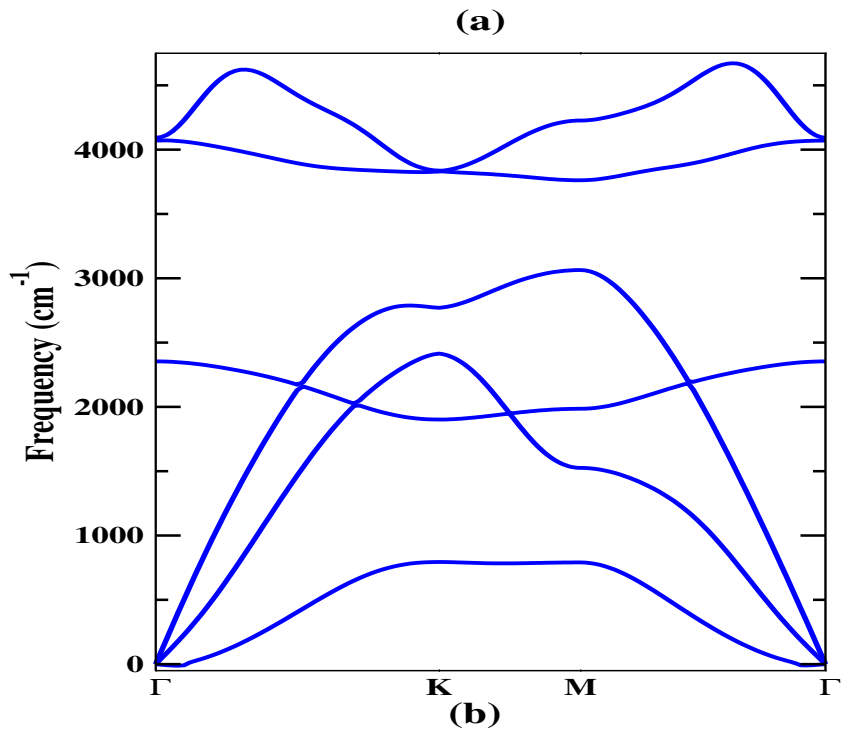

are only two significant independent elastic stiffness coefficients [51]. Another calculated elastic constant, $\mathrm{C}_{66}$ is $\left(\mathrm{C}_{11^{-}}\right.$ $\left.\mathrm{C}_{12}\right) / 2$. The in-plane stiffness $(\mathrm{C})$ and Poisson's ratio $(\vartheta)$ are represented by $C_{11} \times\left[1-\left(C_{12} / \mathrm{C}_{11}\right)^{2}\right]$ and $C_{12} / C_{11}$, respectively. In $2 \mathrm{D}$ materials, the bulk modulus which gives a measure of the material's resilience to an external biaxial strain is calculated in terms of layer modulus $(\gamma)$ with the expression, $\gamma=\left(\mathrm{C}_{11}+\mathrm{C}_{22}+2 \mathrm{C}_{12}\right) / 4$. In addition, using these second-order elastic constants $\mathrm{C}_{i j}$, the shear modulus, $\mathrm{K}=\mathrm{C} / 2(1-\vartheta)$, is calculated. The shear modulus estimates the resistance of the material to the shear strain. Further, the in-plane stiffness and Poisson's ratio are used to test the mechanical anisotropy. They are functions of the direction angles $\theta$, which can be represented as [52]:

$$
\begin{aligned}
C(\theta) & =\frac{C_{11} C_{22}-C_{12}^{2}}{C_{22} \cos ^{4}(\theta)+A \cos ^{2}(\theta) \sin ^{2}(\theta)+C_{11} \cos ^{4}(\theta)} \\
\vartheta(\theta) & =\frac{C_{12} \cos ^{4}(\theta)-B \cos ^{2}(\theta) \sin ^{2}(\theta)+C_{12} \cos ^{4}(\theta)}{C_{22} \cos ^{4}(\theta)+A \cos ^{2}(\theta) \sin ^{2}(\theta)+C_{11} \cos ^{4}(\theta)}
\end{aligned}
$$

where $A=\left(C_{11} C_{22}-C_{12}^{2}\right) / C_{66}-2 C_{12}$ and $B=C_{11}+$ $C_{22}-\left(C_{11} C_{22}-C_{12}^{2}\right) / C_{66}$.

The mechanical stability criteria [53] i.e. $\left|C_{11}\right|>\left|C_{12}\right|$ is approved by the FBNF monolayer, indicating the mechanical stability. Comparatively, the computed elastic parameter $\mathrm{C}_{i j}$ of FBNF monolayer are relatively smaller (Table 2). The in-plane stiffness of the FBNF is $168.33 \mathrm{~N} / \mathrm{m}$, less than h-BN (266.29 N/m ) indicating the softening. Similarly, the Poisson's ratio of FBNF is 0.17 , also not more than h-BN (0.21) but comparable to graphene [18] indicating reduction in sensitivity toward uniaxial strain. Similarly, the shear $(\mathrm{K})$ and layer moduli $(\gamma)$ are $71.70 \mathrm{~N} / \mathrm{m}$ and $110.88 \mathrm{~N} / \mathrm{m}$, respectively. These values are also smaller than h-BN indicating the less resilient material. The physics behind the decrease in the value of mechanical parameter after fluorination in the h-BN (FBNF) is attributed to the decrease in cohesive energy per atom and elongation in $\mathrm{B}-\mathrm{N}$ covalent bond. Further, the directional dependency of in-plane stiffness and Poisson's ratio is checked by polar plot fit using Equ. 5. From the polar plot shown in Fig. 5, the Poisson ratio and in-plane stiffness for FBNF is a 
Table 2

The calculated elastic constants $C_{i j}$, in-plane stiffness $\mathrm{C}$, and Poissons ratio $\vartheta$, shear modulus $\mathrm{K}$ and layer modulus $\gamma$ for each MLs

\begin{tabular}{|c|c|c|c|c|c|c|}
\hline \multirow[t]{2}{*}{ Monolayer } & \multicolumn{2}{|c|}{$\mathrm{C}_{i j}(\mathrm{~N} / \mathrm{m})$} & \multirow{2}{*}{$\mathrm{C}(\mathrm{N} / \mathrm{m})$} & \multirow[t]{2}{*}{$\vartheta$} & \multirow[t]{2}{*}{$\mathrm{K}(\mathrm{N} / \mathrm{m})$} & \multirow[t]{2}{*}{$\gamma(\mathrm{N} / \mathrm{m})$} \\
\hline & $\mathrm{C}_{11}$ & $\mathrm{C}_{12}$ & & & & \\
\hline h-BN & 278.45 & 58.19 & 266.29 & 0.21 & 110.13 & 168.33 \\
\hline Exp.[55] & 270.0 & 56.2 & 258.3 & 0.21 & 106.9 & 163.1 \\
\hline FBNF & 173.58 & 30.18 & 168.33 & 0.17 & 71.70 & 110.88 \\
\hline Ref.[56] & 188.64 & 35.02 & - & - & - & - \\
\hline
\end{tabular}
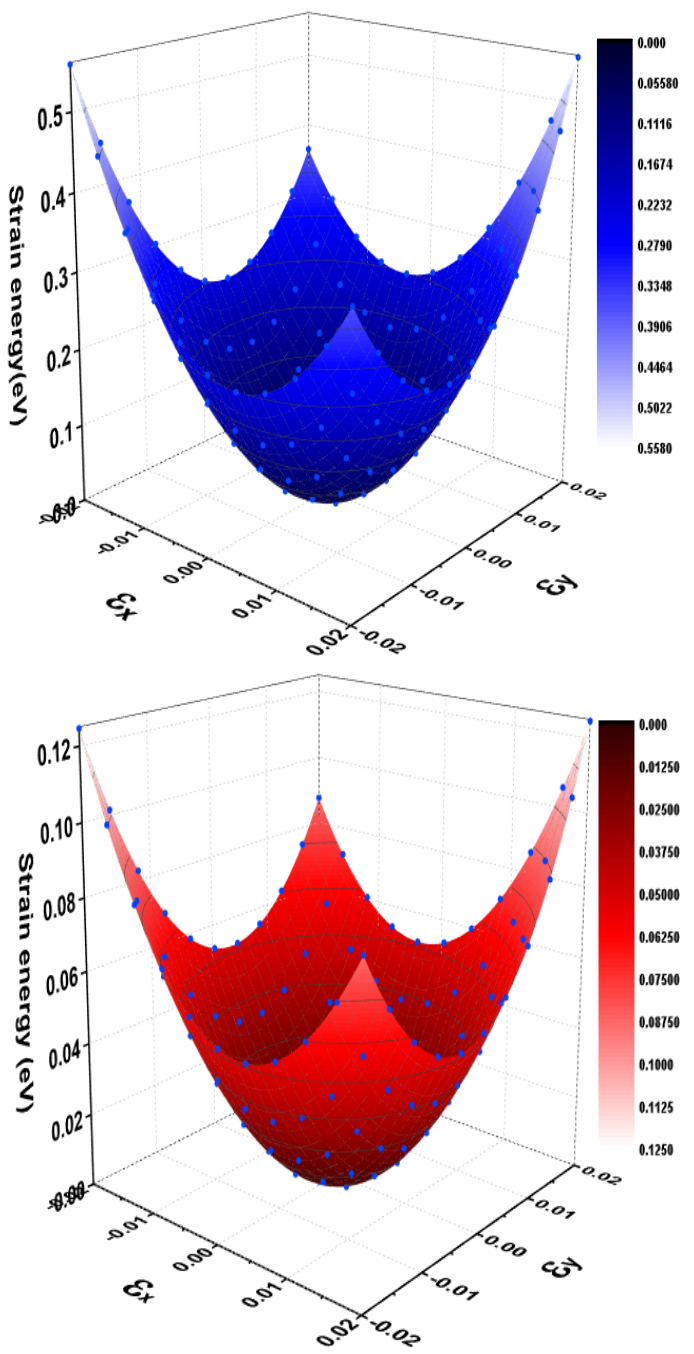

Figure 4: (Color online) Three-dimensional surface plot of strain energy versus strain for h-BN (blue) and FBNF (red) monolayers.

perfect circle, similar to h-BN, indicates elastic isotropy. The computed mechanical parameters are very close to both experimental and theoretical reports (Table 2).

It's worth in mentioning that similar results are computed by previous report in hydrogen passivated hexagonal $\mathrm{GeC}$ monolayer[54].
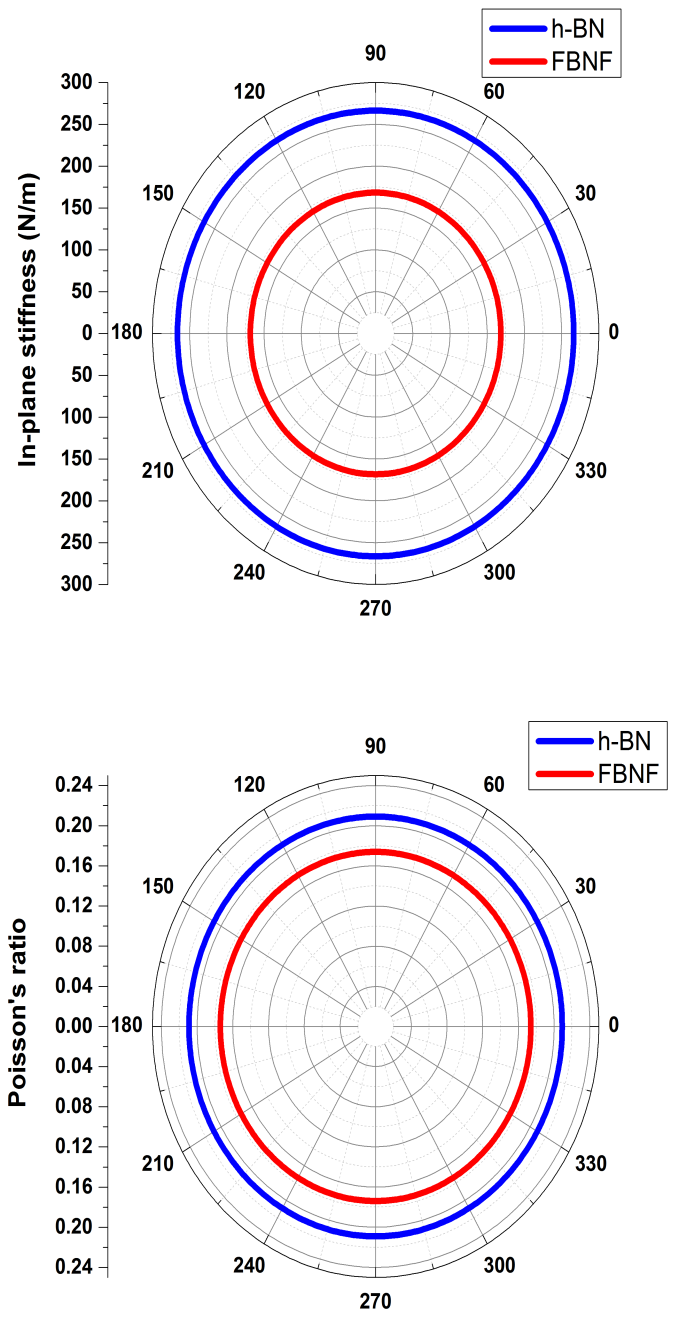

Figure 5: (Color online) The polar plot of orientation dependent in-plane stifness and Poisson's ratio for h-BN and FBNF monolayers.

\subsection{Electronic properties}

We calculate the spin-independent band structure and partial density of states (PDOS) for FBNF and compare it with h-BN. The electronic band structure is calculated along the $\Gamma-\mathrm{K}-\mathrm{M}-\Gamma$ direction of the Brillouin Zone (BZ) ( Fig. 6). The valence band maximum (VBM ) and conduction band minimum $(\mathrm{CBM})$ is positioned at the $\Gamma$ point of $\mathrm{BZ}$ 
(a)

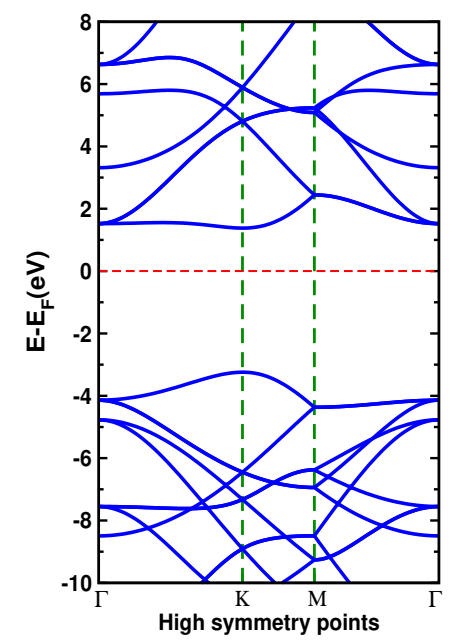

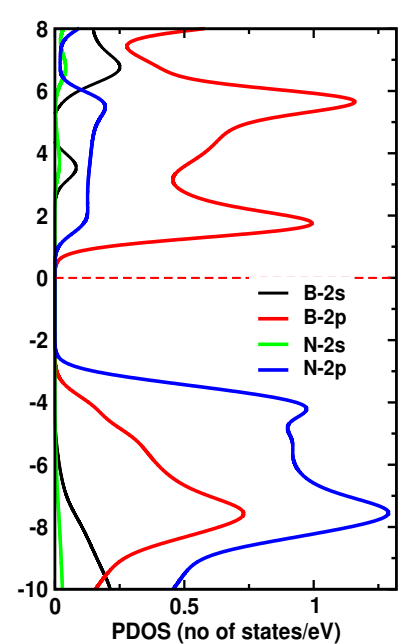

(b)
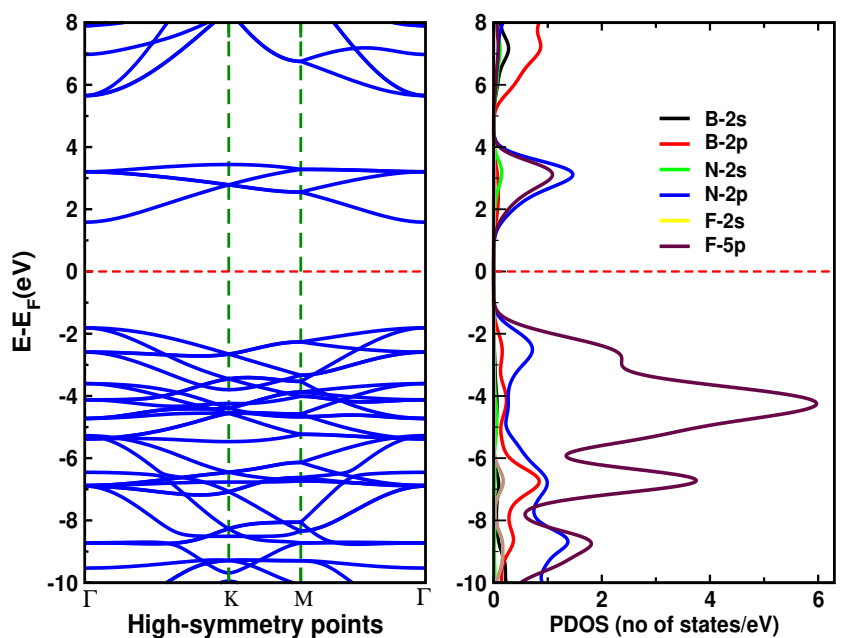

Figure 6: (Color online) The electronic band structures and partial density of states (PDOS) for (a) h-BN (b) FBNF monolayers

with bandgap $3.39 \mathrm{eV}$ indicates the FBNF a large bandgap semiconductor agrees with the previous report[29]. The computed bandgap is $26.62 \%$ smaller than h-BN $(4.62$ $\mathrm{eV})$. The $\mathrm{N}-2 p$ and $\mathrm{F}-5 p, \mathrm{~B}-2 p$ energy orbitals dominate the contribution in CBM and VBM, respectively in the vicinity of Fermi level to reduce the bandgap relative to h-BN. This also demonstrates the influence of fluorination. This wide direct bandgap and induced semiconducting functionality of FBNF makes it a potential candidate material for application in high-temperature processors, solid-state lighting, solar cells, and power electronics[57, 58].

\subsection{Optical properties}

After making a good understanding of electronic properties, which are inherently connected to the optical behavior of a semiconductor (Equ. 4), it is interesting to discuss the optical behavior of FBNF monolayer. The optical response is calculated by analyzing the amplitude and number of peaks in the optical response curves with the photon energy range of $0 \mathrm{eV}$ to $25 \mathrm{eV}$. The incident polarized light is considered in in-plane $(\mathrm{E} \| \mathrm{x})$, and out-of-plane $(\mathrm{E} \| \mathrm{z})$ directions, which are sufficient directions to describe the optical behavior of this 2D system. The spin non-polarized calculations is performed to investigate the important optical parameters such as the real $\left(\varepsilon_{1}(\omega)\right)$ and imaginary $\left(\varepsilon_{2}(\omega)\right)$ parts of dielectric functions, absorption coefficient $(\alpha(\omega))$, electron energy loss function (ELF) $(\mathrm{L}(\omega))$, refractive index $(\eta(\omega))$, and reflectivity $(R(\omega))$. To make a comparison, these optical parameters are plotted side by side (Fig. 7-12) for both directions and also compared with the pristine h-BN.

The real part of the dielectric function $\left(\varepsilon_{1}(w)\right)$ is related to the energy stored by the medium when a material is exposed to an electromagnetic spectrum. The optical spectra in $\varepsilon_{1}(\omega)$ (Fig. 7) for FBNF mainly arise approximately in between $4.6 \mathrm{eV}$ to $15.2 \mathrm{eV}$ with the early sharpest peaks at $8.79 \mathrm{eV}$ and $12.55 \mathrm{eV}$ in $\mathrm{E} \| \mathrm{x}$. Meanwhile, such sharpest peaks occur at $7.71 \mathrm{eV}$ and $11.59 \mathrm{eV}$ in $\mathrm{E} \| \mathrm{z}$, significantly different from $\mathrm{h}-\mathrm{BN}$. The value of $\varepsilon_{1}(\omega)$ is positive throughout the whole energy range in the in-plane direction but a negative value is detected in between the $11.95 \mathrm{eV}$ to $12.45 \mathrm{eV}$ for out-of-plane direction in FBNF monolayer which is unprecedented in h-BN. The corresponding negative frequency is known as plasma frequency $\left(\omega_{p}\right)$, which is very important to understand many exotic optical phenomena[59]. The static dielectric function $\left(\varepsilon_{1}(0)\right)$, the value of $\varepsilon_{1}(\omega)$ at zero photon energy, is $1.5(\approx \mathrm{h}-\mathrm{BN})$ for inplane direction but higher (1.65) in out-of-plane direction.

The major optical behavior of a material is linked with the imaginary part of dielectric function $\left(\varepsilon_{2}(\omega)\right)$ (Eqns. (3$8)$ ). The $\varepsilon_{2}(\omega)$ describes the inter-band transitions by analyzing the major peaks. All the optical inter-band transitions are essentially due to the $p$ orbitals of $\mathrm{F}, \mathrm{B}$, and $\mathrm{N}$ atoms which can be described by analyzing PDOS (Fig. 6). The major sharper peaks (Fig. 8) arise at $9.16 \mathrm{eV}$ and $9.95 \mathrm{eV}$ ( blue-shifted) in $\mathrm{E} \| \mathrm{x}$, and $7.95 \mathrm{eV}, 8.59 \mathrm{eV}$ and $11.88 \mathrm{eV}$ (red-shifted) in $\mathrm{E} \| \mathrm{z}$ displays earlier interband trasitions in FBNF. The higher number and larger intensity in spectra indicates the multiple inter-band transitions and enhanced optical response of FBNF than the pristine counterpart.

The electron energy loss function (ELF) describes the amount of energy loss through the material upon the incidence of photon energy. The multiple energy losses in the energy range $4.25 \mathrm{eV}$ to $25.00 \mathrm{eV}$ (Fig. 9) is detected in FBNF for both directions. The major energy loss peaks arises at $9.29 \mathrm{eV}, 10.19 \mathrm{eV}, 13.88 \mathrm{eV}, 15.23 \mathrm{eV}$ and at $8.83 \mathrm{eV}, 12.47 \mathrm{eV}, 15.87 \mathrm{eV}, 18.0 \mathrm{eV}, 19.40 \mathrm{eV}$ for FBNF in in-plane and out-of-plane direction, respectively. The energy loss is significantly lower in FBNF than the pristine counterpart in the in-plane direction. The sharpest resonance peak at $12.47 \mathrm{eV}$ due to plasma frequency $\left(\omega_{p}\right)$ creates significant energy loss. The energy loss is highly anisotropic like other paramters in FBNF as well as h-BN. 


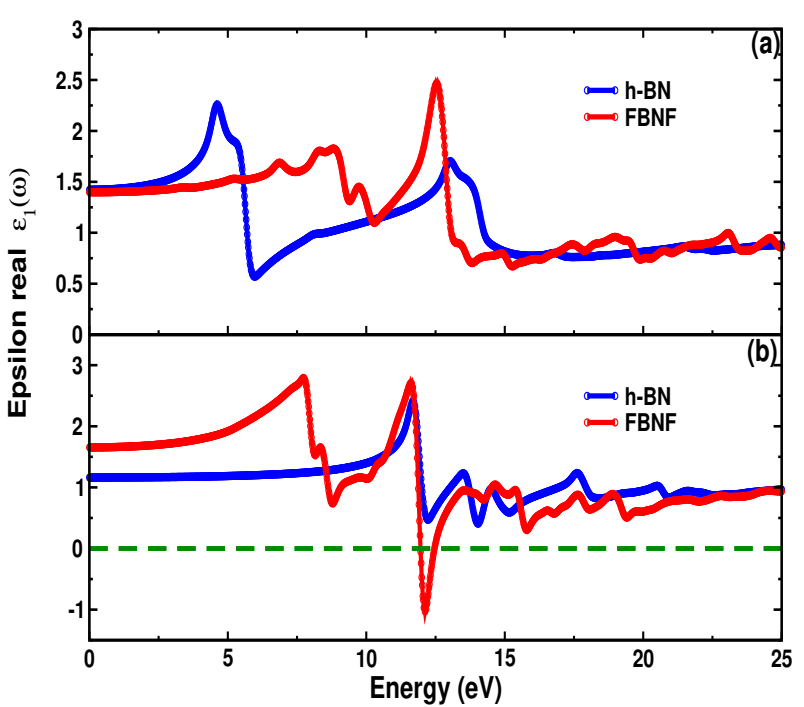

Figure 7: (Color online) Real parts of dielectric function for h-BN and FBNF in (a) in-plane, and (b) out-of-plane direction of electric field polarizations

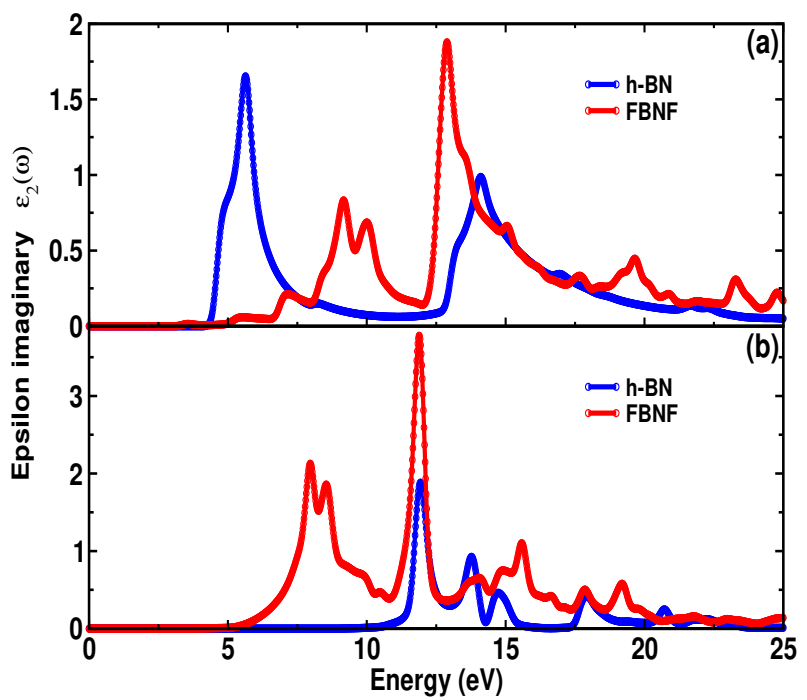

Figure 8: (Color online) Imaginary parts of dielectric function for h-BN and FBNF in (a) in-plane, and (b) out-of-plane direction of electric field polarization

The absorption coefficient $\alpha(\omega)$ spectra describe the light-harvesting quality of the material. The absorption edges $\left(A_{e}\right)$, the threshold value of energy absorption spectra, are at $6.71 \mathrm{eV}$ (red-shifted) and at $5.49 \mathrm{eV}$ (blue-shifted) for in-plane and out-of-plane direction (Fig. 10), respectively. Null absorption in infrared region (IR) and visible region (VR) for FBNF makes it a perfect material in optical fibers and beam splitters[60]. The highest absorption peak is observed at $12.91 \mathrm{eV}\left(\approx 9.01 \times 10^{6} \mathrm{~cm}^{-1}\right)$ and $12.23 \mathrm{eV}$ for in-plane and out-plane direction which is significantly better than pristine h-BN. The exceptional long-range absorbance, from $6.71 \mathrm{eV}$ to $25 \mathrm{eV}$, and multiple absorption peaks in the ultraviolet region (UV) region make FBNF monolayer an appropriate light-harvesting material. Additionally, the

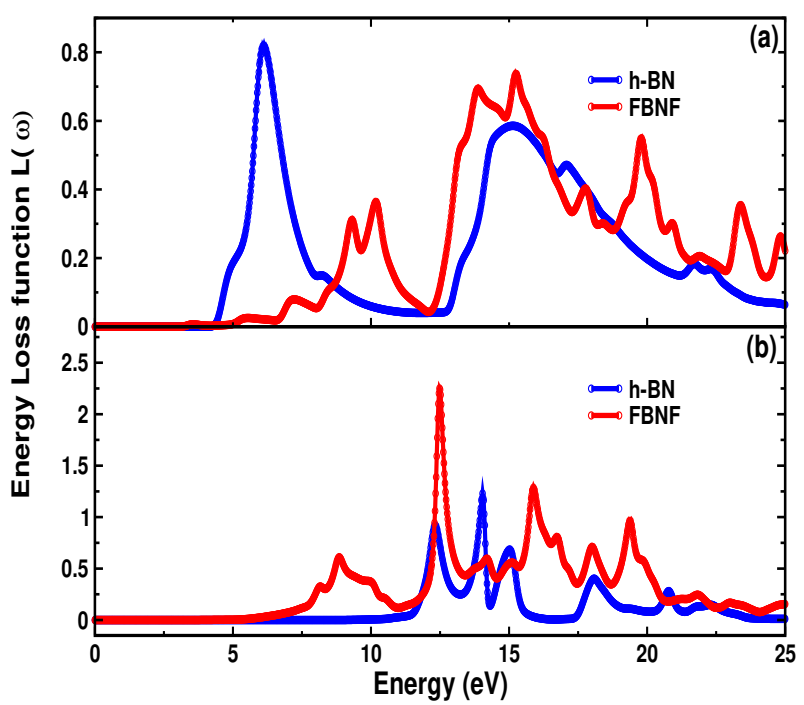

Figure 9: (Color online) Electron energy loss function(ELF) for $\mathrm{h}$-BN and FBNF in (a) in-plane, and (b) out-of-plane direction of electric field polarization.

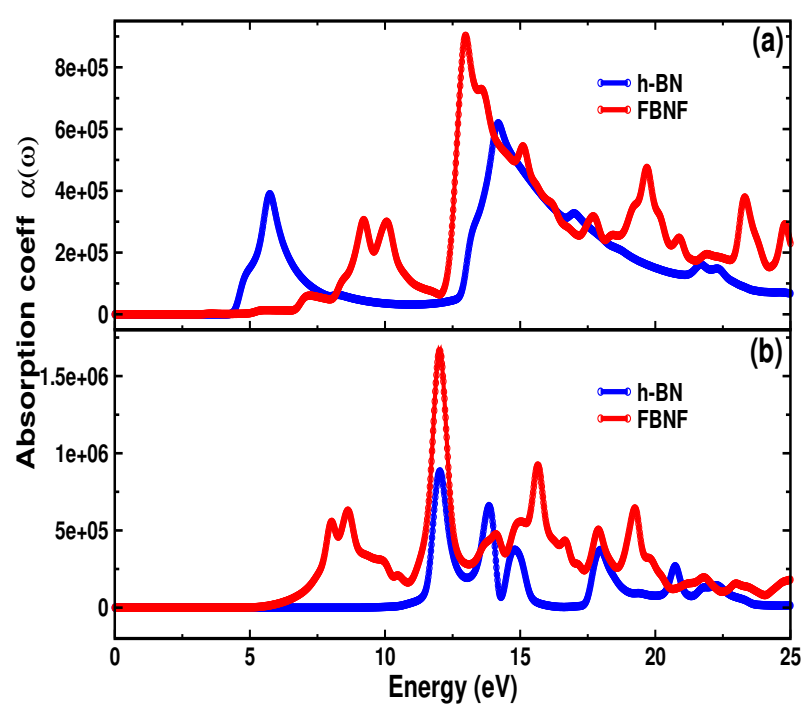

Figure 10: (Color online) Absorption coefficient for h-BN and FBNF in (a) in-plane, and (b) out-of-plane direction of electric field polarization.

strong optical anisotropy broads its applications in light polarizers and wave guides materials.

Besides, multiple reflection sharp peaks (Fig. 11) within the same range of absorption coefficient peaks are detected in both directions. The maximum reflectivity is $10 \%(7.95$ $\mathrm{eV})$ and $40 \%(12.29 \mathrm{eV})$ for in-plane and out-plane direction which is higher than h-BN. The abrupt rise of reflectivity at $12.29 \mathrm{eV}$ in out-of-plane direction is connected to the plasma frequency and the corresponding metallicity. The loss of oscillatory reflectivity curve beyond $20 \mathrm{eV}$ indicates the monolayer's transparency region.

The refractive index of a material describes the behavior of light propagating through it (Fig.12). The static refractive index $(\eta(0))$, the value of the refractive index at zero energy 
Table 3

Static dielectric constant $\varepsilon_{1}(0)$ and static refractive index $\eta(0)$ of $\mathrm{h}-\mathrm{BN}$ and FBNF monolayers for $\mathrm{E} \| \mathrm{x}$ and $\mathrm{E} \| \mathrm{z}$ polarization

\begin{tabular}{|c|c|c|c|c|c|c|c|}
\hline \multirow[t]{2}{*}{ Monolayer } & \multirow[t]{2}{*}{ Methods } & \multicolumn{2}{|c|}{$\eta(0)$} & \multicolumn{2}{|c|}{$\varepsilon_{1}(0)$} & \multicolumn{2}{|c|}{$\mathrm{A}_{e}$} \\
\hline & & $E \| x$ & $E \| z$ & $E \| x$ & $E \| z$ & $E \| x$ & $E \| z$ \\
\hline \multirow[t]{3}{*}{ h-BN } & GGA & 1.18 & 1.08 & 1.42 & 1.16 & 3.97 & 8.67 \\
\hline & GGA & $1.17[62]$ & - & $1.37[62]$ & - & - & - \\
\hline & LDA & - & - & $2.19[61]$ & $1.50[61]$ & - & - \\
\hline FBNF & GGA & 1.19 & 1.29 & 1.40 & 1.65 & 2.89 & 3.04 \\
\hline
\end{tabular}

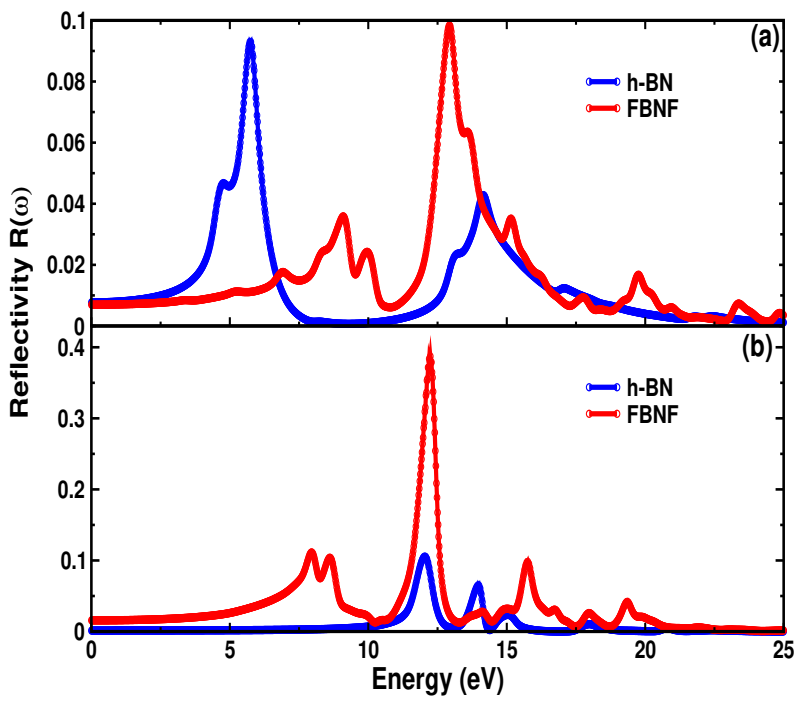

Figure 11: (Color online) Reflectivity for $\mathrm{h}-\mathrm{BN}$ and FBNF in (a) in-plane, and (b) out-of-plane direction of electric field polarization.

is 1.08 and 1.29 for in-plane and out-of-plane direction which is higher than h-BN. However, the highest value of the refractive index is 1.60 (at $12.55 \mathrm{eV}$ ) and 1.77 (at $11.67 \mathrm{eV}$ ) for FBNF in in-plane and out-of-plane directions. The findings of the optical calculation (Table 3 ) are very consistent with the available literature [61, 62].

With a careful analysis, it is clear that the induced large bandgap of FBNF tends to create the optical spectra absent in the IR, and in the VR, and only appear around the UV regions from $4.61 \mathrm{eV}$ to $22.10 \mathrm{eV}$. The multiple oscillatory peaks in the real and imaginary dielectric curve demonstrate the rigorous inter-band transitions. This supports FBNF to possess the highest absorbance (up to $9.01 \times 10^{5} \mathrm{~cm}^{-1}$ ) with small electron energy loss in in-plane direction demonstrating an outstanding optical response. Plus, the strong optical anisotropy enriches its optical quality to make it a proper optoelectronic material. It is worth mentioning that, similar optical behavior is observed in the fluorinated $\mathrm{SnC}$ monolayer calculated by using first-principle DFT calculations[63].

\section{Conclusions}

In summary, The FBNF is found to be a structurally, mechanically, and dynamically stable monolayer. The $\mathrm{sp}^{3}$

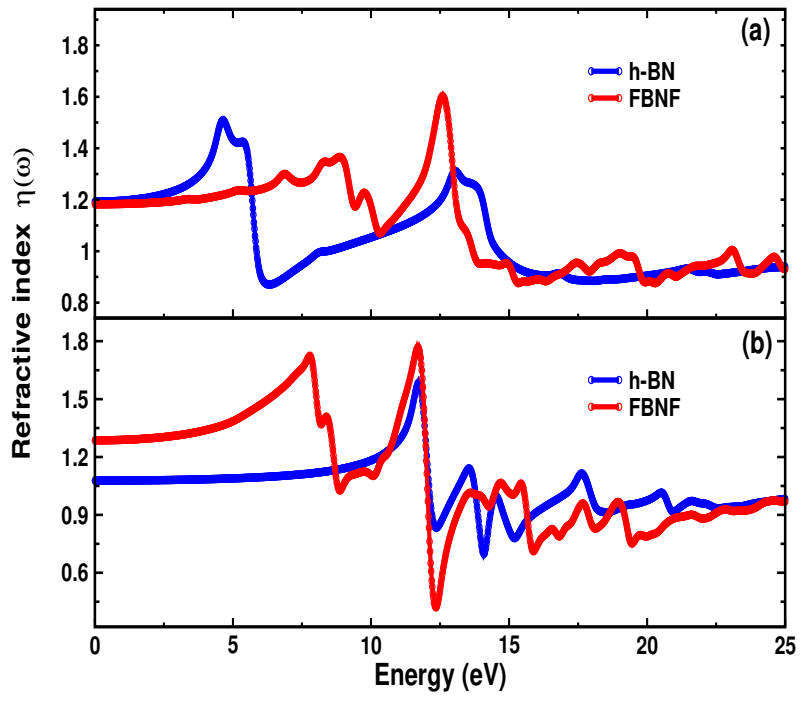

Figure 12: (Color online) Reflectivity for h-BN and FBNF in (a) in-plane, and (b) out-of-plane direction of electric field polarization.

hybridized $\mathrm{B}-\mathrm{N}$ in FBNF, is longer than in $\mathrm{h}-\mathrm{BN}$ is involved in buckling to soften the structure by retaining the structural and elastic isotropy. The F-5 $p$ orbital plays a role to create the insulator-semiconductor electronic transition. The visible range PBE-bandgap supports the largest optical absorption, low electron energy loss, and good reflectivity. In addition, the strong optical anisotropy enriches the optical quality and establishes this material as a proper candidate in optoelectronic devices. These outstanding findings are also supported by the experimental evidence [31]. Hence, the FBNF monolayer is a potential candidate for nanomechanical and optoelectronic device applications.

\section{Acknowledgment}

This research was supported in part through computational resources provided by the Kathmandu University Supercomputer Centre, which was established with equipment donated by CERN. Shambhu is very grateful to the Supercomputer Center (Kathmandu University) for providing high-performance computing facilities during the research work. A fruitful discussion with Dr. Rajendra Adhikari and Keshav Sigdel (Kathmandu University) is acknowledged. 
Persistent family supports from sisters Sita/Sarita Bhandari is also acknowledged.

\section{References}

[1] Kostya S. Novoselov, Andre K. Geim, Sergei Vladimirovich Morozov, Dingde Jiang, Michail I. Katsnelson, IVa Grigorieva, SVb Dubonos, Firsov, and AA. Two-dimensional gas of massless Dirac fermions in graphene. nature, 438(7065):197-200, 2005.

[2] Qing Peng, Wei Ji, and Suvranu De. Mechanical properties of the hexagonal boron nitride monolayer: Ab initio study. Computational Materials Science, 56:11-17, 2012.

[3] Li Song, Lijie Ci, Hao Lu, Pavel B. Sorokin, Chuanhong Jin, Jie Ni, Alexander G. Kvashnin, Dmitry G. Kvashnin, Jun Lou, Boris I. Yakobson, et al. Large scale growth and characterization of atomic hexagonal boron nitride layers. Nano letters, 10(8):3209-3215, 2010.

[4] Shambhu Bhandari Sharma, Ramchandra Bhatta, Keshav Raj Sigdel, Rajendra P Adhikari, and Gopi Chandra Kaphle. Structural, electronic, magnetic and mechanical properties of vanadium-doped boron nitride monolayer. The European Physical Journal B, 94(6):1-7, 2021.

[5] Zhiming Shi, Zhuhua Zhang, Alex Kutana, and Boris I. Yakobson. Predicting two-dimensional silicon carbide monolayers. ACS nano, 9 (10):9802-9809, 2015.

[6] Saptarshi Das, Marcel Demarteau, and Andreas Roelofs. Ambipolar phosphorene field effect transistor. ACS nano, 8(11):11730-11738, 2014.

[7] Kin Fai Mak, Changgu Lee, James Hone, Jie Shan, and Tony F Heinz. Atomically thin mos 2: a new direct-gap semiconductor. Physical review letters, 105(13):136805, 2010.

[8] Anjna Devi, Arun Kumar, Tanuj Kumar, Rajendra Adhikari, PK Ahluwalia, Amarjeet Singh, et al. Structural, electronic and magnetic properties of crmsn and crmsen nanoflakes: An ab-initio investigation. Physica E: Low-dimensional Systems and Nanostructures, page 114825, 2021.

[9] Jin-Cheng Lei, Xu Zhang, and Zhen Zhou. Recent advances in mxene: Preparation, properties, and applications. Frontiers of Physics, 10(3):276-286, 2015.

[10] Kailiang Zhang, Yulin Feng, Fang Wang, Zhengchun Yang, and John Wang. Two dimensional hexagonal boron nitride (2d-hbn): synthesis, properties and applications. Journal of Materials Chemistry C, 5(46): 11992-12022, 2017.

[11] Lu Hua Li, Ying Chen, Gavin Behan, Hongzhou Zhang, Mladen Petravic, and Alexey M Glushenkov. Large-scale mechanical peeling of boron nitride nanosheets by low-energy ball milling. Journal of materials chemistry, 21(32):11862-11866, 2011.

[12] Chuanhong Jin, Fang Lin, Kazu Suenaga, and Sumio Iijima. Fabrication of a freestanding boron nitride single layer and its defect assignments. Physical review letters, 102(19): 195505, 2009.

[13] Wei-Qiang Han, Lijun Wu, Yimei Zhu, Kenji Watanabe, and Takashi Taniguchi. Structure of chemically derived mono-and few-atomiclayer boron nitride sheets. Applied Physics Letters, 93(22):223103, 2008.

[14] Chunyi Zhi, Yoshio Bando, Chengchun Tang, Hiroaki Kuwahara, and Dimitri Golberg. Large-scale fabrication of boron nitride nanosheets and their utilization in polymeric composites with improved thermal and mechanical properties. Advanced Materials, 21(28):2889-2893, 2009.

[15] Laurence Vel, Gerard Demazeau, and Jean Etourneau. Cubic boron nitride: synthesis, physicochemical properties and applications. Materials Science and Engineering: B, 10(2):149-164, 1991.

[16] Sen Lin, Xinxin Ye, Ryan S Johnson, and Hua Guo. First-principles investigations of metal (cu, ag, au, pt, rh, pd, fe, co, and ir) doped hexagonal boron nitride nanosheets: stability and catalysis of co oxidation. The Journal of Physical Chemistry C, 117(33):1731917326, 2013.

[17] Jonathan da Rocha Martins and Helio Chacham. Disorder and segregation in b- c- $\mathrm{n}$ graphene-type layers and nanotubes: tuning the band gap. Acs Nano, 5(1):385-393, 2011.

[18] Richard Charles Andrew, Refilwe Edwin Mapasha, Aniekan M Ukpong, and Nithaya Chetty. Mechanical properties of graphene and boronitrene. Physical review B, 85(12):125428, 2012.

[19] Qiran Cai, Declan Scullion, Wei Gan, Alexey Falin, Shunying Zhang, Kenji Watanabe, Takashi Taniguchi, Ying Chen, Elton JG Santos, and $\mathrm{Lu} \mathrm{Hua} \mathrm{Li}$. High thermal conductivity of high-quality monolayer boron nitride and its thermal expansion. Science advances, 5(6): eaav0129, 2019.

[20] Cory R Dean, Andrea F Young, Inanc Meric, Chris Lee, Lei Wang, Sebastian Sorgenfrei, Kenji Watanabe, Takashi Taniguchi, Phillip Kim, Kenneth L Shepard, et al. Boron nitride substrates for highquality graphene electronics. Nature nanotechnology, 5(10):722726, 2010.

[21] Kazuhisa Miyoshi, Donald H Buckley, John J Pouch, Samuel A Alterovitz, and Harold E Sliney. Mechanical strength and tribological behavior of ion-beam-deposited boron nitride films on non-metallic substrates. Surface and Coatings Technology, 33:221-233, 1987.

[22] Si Qin, Weiwei Lei, Dan Liu, and Ying Chen. Advanced n-doped mesoporous molybdenum disulfide nanosheets and the enhanced lithium-ion storage performance. Journal of Materials Chemistry A, 4(4):1440-1445, 2016.

[23] R MâĂŹnassri. Chniba boudjada n and cheikhrouhou a. J. Alloys Compd., 2015:626, 2015.

[24] M Wang, S Tang, J Ren, B Wang, Y Han, and Y Dai. Magnetism in boron nitride monolayer induced by cobalt or nickel doping. Journal of Superconductivity and Novel Magnetism, 31(5):1559-1565, 2018.

[25] YaLei Bai, MinMin Xue, ZhiQiang Zhao, and ZhuHua Zhang. Functionalizations of boron nitride nanostructures. Science China Technological Sciences, 64:1-10, 2021.

[26] Yanli Wang, Yi Ding, and Jun Ni. Fluorination-induced halfmetallicity in zigzag boron nitride nanoribbons: First-principles calculations. Physical Review B, 81(19):193407, 2010.

[27] Jian Zhou, Qian Wang, Qiang Sun, and Puru Jena. Electronic and magnetic properties of a bn sheet decorated with hydrogen and fluorine. Physical Review B, 81(8):085442, 2010.

[28] Shaobin Tang, Jianping Yu, and Liangxian Liu. Tunable doping and band gap of graphene on functionalized hexagonal boron nitride with hydrogen and fluorine. Physical Chemistry Chemical Physics, 15 (14):5067-5077, 2013.

[29] Mohammad Noor-A-Alam, Hye Jung Kim, and Young-Han Shin. Dipolar polarization and piezoelectricity of a hexagonal boron nitride sheet decorated with hydrogen and fluorine. Physical Chemistry Chemical Physics, 16(14):6575-6582, 2014.

[30] Sruthi Radhakrishnan, Deya Das, Atanu Samanta, A Carlos, Liangzi Deng, Lawrence B Alemany, Thomas K Weldeghiorghis, Valery N Khabashesku, Vidya Kochat, Zehua Jin, et al. Fluorinated h-bn as a magnetic semiconductor. Science advances, 3(7):e1700842, 2017.

[31] Vishnu S Surendran, B Bindhu, and CR Indulal. Enhancement of the optical properties of fluorinated boron nitride nanosheets. In AIP Conference Proceedings, volume 2220, page 020016. AIP Publishing LLC, 2020.

[32] José M Soler, Emilio Artacho, Julian D Gale, Alberto García, Javier Junquera, Pablo Ordejón, and Daniel Sánchez-Portal. The siesta method for ab initio order-n materials simulation. Journal of Physics: Condensed Matter, 14(11):2745, 2002.

[33] Emilio Artacho, Daniel Sánchez-Portal, Pablo Ordejón, Alberto Garcia, and José M Soler. Linear-scaling ab-initio calculations for large and complex systems. physica status solidi (b), 215(1):809-817, 1999.

[34] Norman Troullier and José Luís Martins. Efficient pseudopotentials for plane-wave calculations. Physical review B, 43(3):1993, 1991.

[35] John P Perdew, Kieron Burke, and Matthias Ernzerhof. Generalized gradient approximation made simple. Physical review letters, 77(18): $3865,1996$.

[36] Hendrik J Monkhorst and James D Pack. Special points for brillouinzone integrations. Physical review B, 13(12):5188, 1976. 
[37] Sonali Saha, TP Sinha, and Abhijit Mookerjee. Electronic structure, chemical bonding, and optical properties of paraelectric batio 3 . Physical Review B, 62(13):8828, 2000.

[38] M Fadaie, N Shahtahmassebi, MR Roknabad, and O Gulseren. Investigation of new two-dimensional materials derived from stanene. Computational Materials Science, 137:208-214, 2017.

[39] Mohammad Ali Mohebpour, Sahar Izadi Vishkayi, and Meysam Bagheri Tagani. Tuning electronic and optical properties of free-standing sn2bi monolayer stabilized by hydrogenation. Journal of Applied Physics, 127(1):014302, 2020.

[40] AZ AlZahrani and GP Srivastava. Structural and electronic properties of h-passivated graphene. Applied surface science, 256(19):5783$5788,2010$.

[41] LB Drissi, K Sadki, M-H Kourra, and M Bousmina. Strainengineering of janus sic monolayer functionalized with $\mathrm{h}$ and $\mathrm{f}$ atoms. Journal of Applied Physics, 123(18):185106, 2018.

[42] YG Zhou, J Xiao-Dong, ZG Wang, Haiyan Y Xiao, Fei Gao, and Xiaotao $\mathrm{T} \mathrm{Zu}$. Electronic and magnetic properties of metal-doped bn sheet: A first-principles study. Physical Chemistry Chemical Physics, 12(27):7588-7592, 2010.

[43] W Paszkowicz, JB Pelka, M Knapp, T Szyszko, and SJAPA Podsiadlo. Lattice parameters and anisotropic thermal expansion of hexagonal boron nitride in the 10-297.5 k temperature range. Applied Physics A, 75(3):431-435, 2002.

[44] Do-Hyun Kim, Hag-Soo Kim, Min Woo Song, Seunghyun Lee, and Sang Yun Lee. Geometric and electronic structures of monolayer hexagonal boron nitride with multi-vacancy. Nano convergence, 4 (1):1-8, 2017.

[45] Rodrigo G Amorim, Xiaoliang Zhong, Saikat Mukhopadhyay, Ravindra Pandey, Alexandre R Rocha, and Shashi P Karna. Strain-and electric field-induced band gap modulation in nitride nanomembranes. Journal of Physics: Condensed Matter, 25(19):195801, 2013.

[46] Hong-Baek Cho, Yoshinori Tokoi, Satoshi Tanaka, Tsuneo Suzuki, Weihua Jiang, Hisayuki Suematsu, Koichi Niihara, and Tadachika Nakayama. Facile orientation of unmodified bn nanosheets in polysiloxane/bn composite films using a high magnetic field. Journal of materials science, 46(7):2318-2323, 2011.

[47] Li Shao, Yan Li, Qingxin Yuan, Mingyu Li, Yinxiao Du, Fanguang Zeng, Pei Ding, and Honggang Ye. Effects of strain on mechanical and electronic properties of borophene. Materials Research Express, 4(4):045020, 2017.

[48] Akhtar Hussain, Hafiz Muhammad Rafique, Muhammad Tayyab, et al. Computational study of be-doped hexagonal boron nitride (hbn): Structural and electronic properties. Computational Condensed Matter, 23:e00474, 2020.

[49] N Ooi, V Rajan, J Gottlieb, Y Catherine, and JB Adams. Structural properties of hexagonal boron nitride. Modelling and Simulation in Materials Science and Engineering, 14(3):515, 2006.

[50] Mehmet Topsakal, Seymur Cahangirov, and Salim Ciraci. The response of mechanical and electronic properties of graphane to the elastic strain. Applied Physics Letters, 96(9):091912, 2010.

[51] Michael N Blonsky, Houlong L Zhuang, Arunima K Singh, and Richard G Hennig. Ab initio prediction of piezoelectricity in twodimensional materials. ACS nano, 9(10):9885-9891, 2015.

[52] Shao-Bo Chen, Zhao-Yi Zeng, Xiang-Rong Chen, and Xing-Xing Yao. Strain-induced electronic structures, mechanical anisotropy, and piezoelectricity of transition-metal dichalcogenide monolayer crs 2 . Journal of Applied Physics, 128(12):125111, 2020.

[53] Max Born and Rama Dhar Misra. On the stability of crystal lattices. iv. In Mathematical Proceedings of the Cambridge Philosophical Society, volume 36, pages 466-478. Cambridge University Press, 1940.

[54] Zihab Sohbatzadeh, Hossein Asnaashari Eivari, and Davoud Vahedi Fakhrabad. Formation energy and some mechanical properties of hydrogenated hexagonal monolayer of gec. Physica B: Condensed Matter, 547:88-91, 2018.

[55] Alexey Bosak, Jorge Serrano, Michael Krisch, Kenji Watanabe, Takashi Taniguchi, and Hisao Kanda. Elasticity of hexagonal boron nitride: Inelastic x-ray scattering measurements. Physical Review B, 73(4):041402, 2006.

[56] Mohammad Noor-A-Alam, Hye Jung Kim, and Young-Han Shin. Dipolar polarization and piezoelectricity of a hexagonal boron nitride sheet decorated with hydrogen and fluorine. Physical Chemistry Chemical Physics, 16(14):6575-6582, 2014.

[57] Meiyong Liao, Thomas Stergiopoulos, Jose Alvarez, Surojit Chattopadhyay, and Guihua Zhang. Wide-bandgap semiconductors: nanostructures, defects, and applications, 2015.

[58] Javad Jalilian, Fariborz Parandin, and Jaafar Jalilian. Tuning of electronic and optical properties in $\mathrm{znx}(\mathrm{x}=\mathrm{o}, \mathrm{s}$, se and te) monolayer: hybrid functional calculations. Chem. Rev. Lett., 2:76-83, 2019.

[59] Shan Guan, Shengyuan A Yang, Liyan Zhu, Junping $\mathrm{Hu}$, and Yugui Yao. Electronic, dielectric and plasmonic properties of twodimensional electride materials x $2 \mathrm{n}(\mathrm{x}=\mathrm{ca}, \mathrm{sr})$ : A first-principles study. Scientific reports, 5(1):1-14, 2015.

[60] Hamad Rahman Jappor and Majeed Ali Habeeb. Optical properties of two-dimensional gas and gase monolayers. Physica E: Lowdimensional Systems and Nanostructures, 101:251-255, 2018.

[61] Razieh Beiranvand and Shahoo Valedbagi. Electronic and optical properties of h-bn nanosheet: A first principles calculation. Diamond and Related Materials, 58:190-195, 2015.

[62] Akshay M Satawara, Gaushiya A Shaikh, Sanjeev K Gupta, and PN Gajjar. Structural, electronic and optical properties of hexagonal boron-nitride (h-bn) monolayer: An ab-initio study. Materials Today: Proceedings, 2020.

[63] Soleyman Majidi, S Mohammad Elahi, Amirhosein Esmailian, and Faramarz Kanjouri. First principle study of electronic and optical properties of full-fluorinated snc nanosheets. Silicon, 10(3):869-877, 2018. 